\title{
Usefulness of Videofluoroscopic Swallow Study in Treacher Collins Syndrome With Cleft Palate: A Case Report
}

\author{
Do-Won Hwang, $\mathrm{MD}^{1}$, Kang-Jae Jung, $\mathrm{MD}^{1}$, Seung-Yeon Kim, $\mathrm{MD}^{2}$, Jae-Hyung Kim, MD, PhD
}

Departments of ${ }^{1}$ Physical Medicine \& Rehabilitation, ${ }^{2}$ Pediatrics,

Eulji University Hospital, Eulji University School of Medicine, Daejeon, Korea

\begin{abstract}
A 3-year-old girl had multiple anomalies compatible with Treacher Collins Syndrome (TCS). From the neonatal period, sucking was poor, making tube feeding necessary. Excessive saliva was retained in the oral cavity. Nasal leakage caused by the cleft palate was observed when she spoke. The initial videofluoroscopic swallow study (VFSS) showed a poor posterior bolus transit and nasopharyngeal regurgitation. A delayed swallow reflex and bolus stasis at the vallecular and pyriform sinuses were recognized. Based on the VFSS findings, the patient underwent palatoplasty at 20 months of age. At approximately 23 months of age, a follow-up VFSS was performed; poor posterior bolus transit, nasopharyngeal regurgitation, and delayed swallow reflex were not observed. Finally, the patient was able to eat ground or chopped foods and solid foods orally. We deem VFSS to be helpful in deciding the appropriate management of dysphagia in TCS.
\end{abstract}

Keywords Fluoroscopy, Cleft palate, Treacher Collins

\section{INTRODUCTION}

Treacher Collins syndrome (TCS) is an autosomal dominant disorder of craniofacial development, which has an incidence of approximately one in 50,000 live births [1]. TCS is characterized by abnormalities of the pinnae that are frequently associated with atresia of the external

Received December 2, 2013; Accepted February 5, 2014

Corresponding author: Jae-Hyung Kim

Department of Physical Medicine and Rehabilitation, Eulji University Hospital, Eulji University School of Medicine, 95 Dunsanse-ro, Seo-gu, Daejeon 302-799, Korea

Tel: +82-42-611-3629, Fax: +82-42-611-3633, E-mail: rehabkjh@hanmail. net

(c) This is an open-access article distributed under the terms of the Creative Commons Attribution Non-Commercial License (http://creativecommons. org/licenses/by-nc/3.0) which permits unrestricted noncommercial use, distribution, and reproduction in any medium, provided the original work is properly cited.

Copyright $\odot 2014$ by Korean Academy of Rehabilitation Medicine auditory canals and anomalies of the middle ear ossicles. As a result, bilateral conductive hearing loss is common along with hypoplasia of the facial bones, particularly the mandible and zygomatic complex; antimongoloid slanting of the palpebral fissures with coloboma of the lower eyelids and a paucity of lid lashes medial to the defect; and finally, cleft palate [2]. Craniofacial anomalies lead to airway obstruction and dysphagia is caused by temporomandibular joint anomaly, cleft palate, mandibular hypoplasia, and glossoptosis. Patients usually suffer from various functional deficits including communication deficits, bilateral conductive hearing loss, and dysphagia [3].

Because feeding and growth are important components of pediatric rehabilitation, it is necessary to perform precise evaluation and appropriate treatment procedures for dysphagia. The objective of the present study was to 
report the role of videofluoroscopic swallow study (VFSS) and to determine the subsequent treatment of dysphagia in TCS based on the VFSS findings.

\section{CASE REPORT}

A baby girl who was delivered by Caesarean section had multiple anomalies compatible with TCS (Fig. 1A, 1B). She received a tracheostomy and endotracheal intubation had been performed. None of her family members had TCS. A facial computed tomography scan showed hypoplasia of the maxilla and hard palate defects (Fig. $2 \mathrm{~A}, 2 \mathrm{~B})$. We confirmed the presence of a Treacher Collins-Franceschetti syndrome 1 (TCOF1) gene mutation by polymerase chain reaction. We confirmatively diagnosed this case as TCS with a de novo frameshift mutation within the TCOF1 gene (c.880del, p.Val294SerfsX122). Her motor development was delayed. She could sit alone at 13 months of age, and could walk alone at 22 months of age. From the neonatal period, sucking was poor, mak- ing tube feeding necessary. Excessive saliva was retained in the oral cavity and considerable drooling and nasal regurgitation of saliva were seen. Nasal leakage caused by the cleft palate was observed when she spoke. She underwent palatoplasty at 20 months of age. We carried out serial VFSSs, before and after surgical treatment (Table 1). Based on the VFSS findings, we performed the appropriate management of dysphagia in TCS. We used a modified VFSS protocol from Logemann's study [4]. The lateral view of the VFSS was obtained with the child in an upright sitting position, after she had been fed bariumcontaining free water or milk, yogurt (thick liquid), rice porridge (soft), and cookies (hard). In the first VFSS, the child swallowed barium-containing milk on her own from a bottle with nipple. In the second and third VFSSs, the patient swallowed a half spoonful of milk and free water. Subsequently, it was increased to gradually increasing volumes of liquid and progressed to solid food. For psychological stability and accurate examination, the child was fed by her parents.
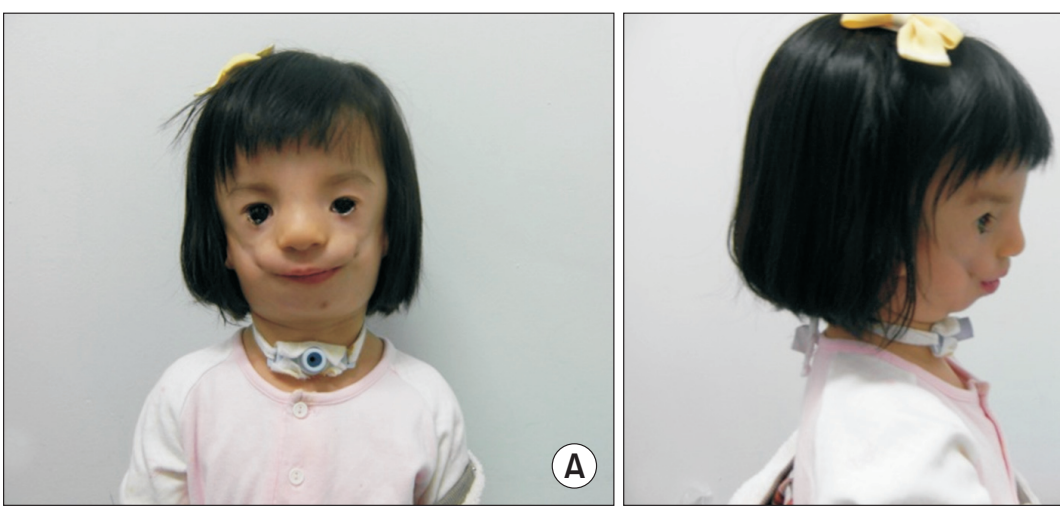

(B)
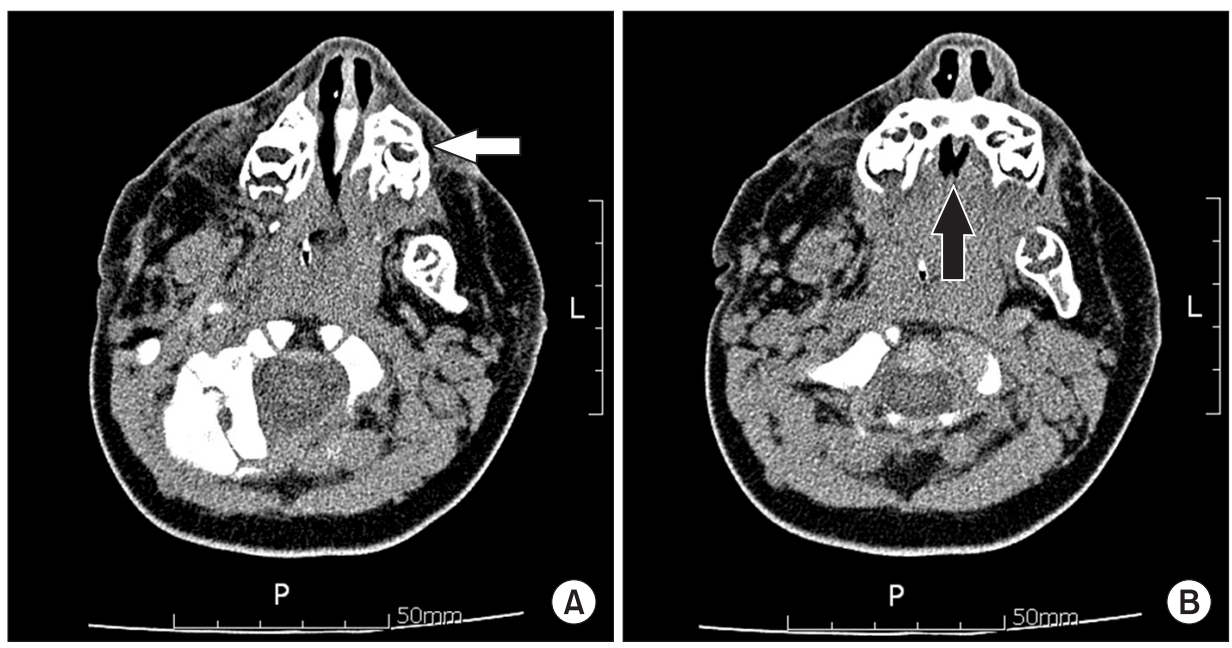

Fig. 1. Clinical features of the patient with facial abnormalities of Treacher Collins syndrome in the frontal (A) and lateral views (B).
Fig. 2. Axial views of facial computed tomography show (A) hypoplasia of the maxilla (white arrow) and (B) hard palate defect (black arrow). 
The first assessment using the VFSS, before surgical repair of the cleft palate

The first VFSS was performed at 12 months of age (Fig. $3 \mathrm{~A}$ ). A delayed oral transit time (OTT, 1.30 seconds), premature bolus loss, material being pushed out with the tongue, and leakage of food or liquid from the mouth were seen during the oral phase. During the pharyngeal phase, we found poor posterior bolus transit (pharyngeal transit time [PTT] 1.94 seconds), a decrease in laryngeal elevation $(0.4 \mathrm{~cm})$, a delayed swallow reflex (pharyngeal delay time [PDT] 0.44 seconds), bolus stasis at the vallecular and pyriform sinuses (grade 1), and nasopharyngeal regurgitation during swallowing a large amount of liquid. Aspiration and penetration were not observed. Based on these findings, tube feeding was discontinued and oral intake was started. We performed dysphagia rehabilitation and recommended surgical reconstruction of the cleft palate.

The second assessment using the VFSS, after surgical repair of the cleft palate

She underwent palatoplasty with the double opposing Z-plasty technique at 20 months of age. After surgical treatment, the second fluoroscopy was performed at 23 months of age (Fig. 3B). Except for poor mastication and delayed OTT (1.50 seconds), lip sealing and tongue base movement were improved during the oral phase compared with those in the previous examination. Poor posterior bolus transit (PTT 1.48 seconds), a decrease in laryngeal elevation $(0.63 \mathrm{~cm})$, and a delayed swallow reflex (PDT 0.44 seconds) persisted during the pharyngeal phase, but nasopharyngeal regurgitation was not observed. Bolus stasis at the vallecular and pyriform sinuses was recognized, but only to a slight degree. We performed an intensive dysphagia rehabilitative program including oromotor facilitation, thermo-tactile stimulation, and re-education of the swallowing muscles. At approximately 24 months of age, the patient could eat ground or chopped foods orally without choking.

The third assessment using the VFSS for the follow-up study

The third VFSS was performed at 31 months of age. It demonstrated an OTT of 1.48 seconds, and lip sealing and tongue movement during the oral phase showed no definite abnormalities. Pharyngeal parameters, such

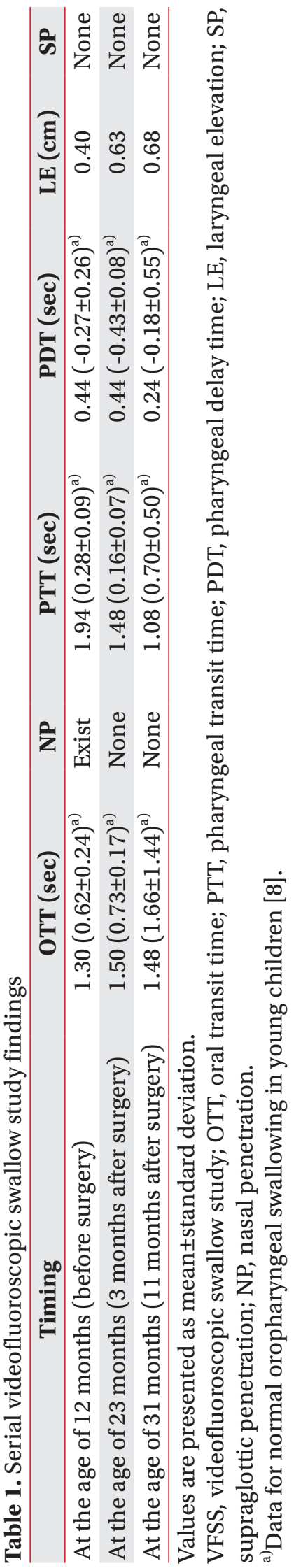

www.e-arm.org 

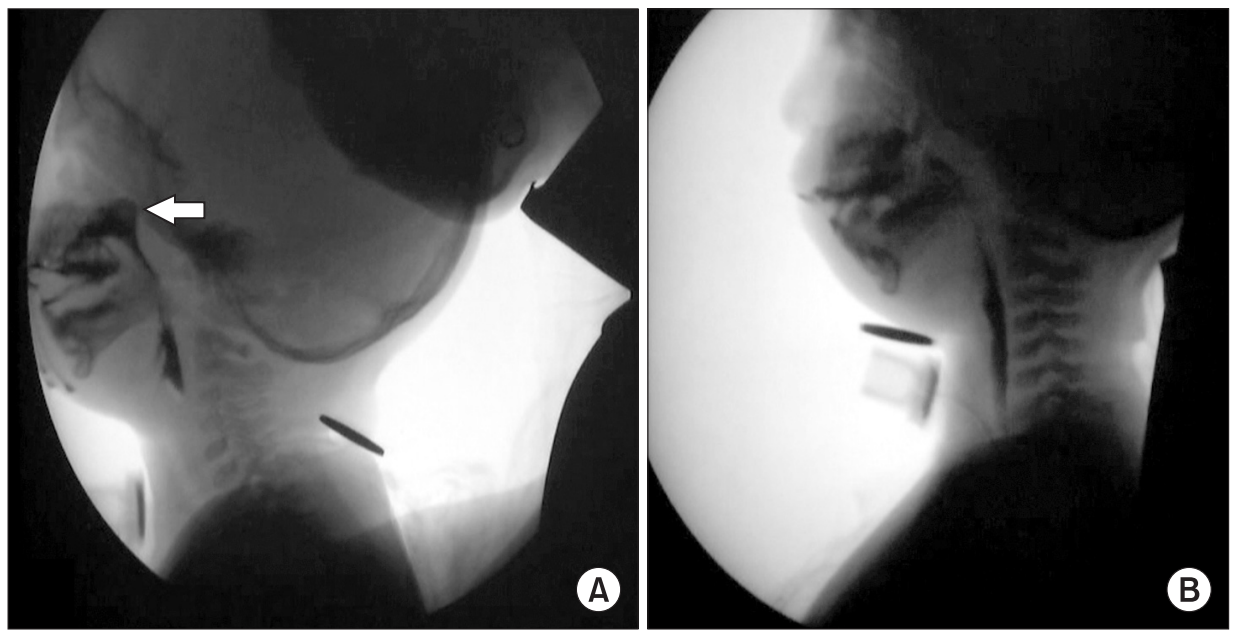

Fig. 3. (A) The first videofluoroscopic swallow study (VFSS) performed before surgical treatment shows nasopharyngeal regurgitation (white arrow) of a large amount of liquid during swallowing and (B) the second VFSS performed after surgical treatment do not show any evidence of nasopharyngeal regurgitation.

as a PTT of 1.08 seconds, a PDT of 0.24 seconds, and a laryngeal elevation of $0.68 \mathrm{~cm}$, were markedly improved compared with those in previous examinations. Nasal regurgitation, supraglottic penetration, and pyriform and vallecular residues were not observed. Eventually, at 36 months of age, the patient could eat solid foods and drink a cup of liquid safely.

\section{DISCUSSION}

Various methods can be used for the evaluation of pediatric dysphagia. Instrumental assessments of swallowing function in pediatric dysphagia include VFSS, upper gastrointestinal study, ultrasonography, radionuclide imaging, flexible endoscopic examination of swallowing (FEES), and cervical auscultation [5]. It is well known that the VFSS is the primary instrumental examination that provides dynamic imaging of oral, pharyngeal, and upper esophageal phases of swallowing. Based on the VFSS findings, we can make an accurate assessment of the swallowing mechanism.

Although VFSS has a risk of radiation exposure, it is a useful tool for the evaluation of dysphagia and allows decision-making regarding the appropriate management of pediatric TCS. After the first VFSS in this research, we performed nasogastric tube removal. We determined the optimal timing for surgical correction of cleft palate based on the VFSS findings, which included delayed OTT, premature bolus loss, poor posterior bolus transit, delayed swallow reflex, mild bolus stasis at the vallecular and pyriform sinuses, and nasopharyngeal regurgitation. Aspiration and penetration were not observed. The patient then received surgical treatment for correction of the cleft palate. We permitted the patient to take only a small amount of liquid and thick liquids with a viscosity of yogurt. Dysphagia rehabilitation including thermotactile stimulation and oromotor facilitation was prescribed. After surgical treatment of the cleft palate, we performed the second VFSS and then recommended that she should be encouraged to drink a small amount of liquid and consume a soft diet, and should receive intensive dysphagia rehabilitation. In this case, the VFSS findings after surgical management were mostly improved compared with the findings before surgical management, except for the OTT. We thought that the OTT was increased due to post-surgical conditions. After the cleft palate repair, nasopharyngeal regurgitation of a large amount of liquid had disappeared. Based on these results, we demonstrated that the cleft palate repair and post-operative dysphagia rehabilitation improved the oral phase function as well as the pharyngeal phase function. Finally, in the third follow-up study, the VFSS findings showed improvements in all oropharyngeal parameters. We then recommended a solid diet and drinking liquid from a cup.

Infants who required tracheostomy are typically decannulated after adequate airway growth and posterior cleft palate repair [6]. In this case, because the patient frequently suffered from sudden choking and asphyxia, she could not be decannulated, and her airway was repaired after a few months by an otolaryngologist.

There are some limitations to this study. Manometric studies permit the quantification of pharyngeal contractile forces, or quantification of the magnitude of intra- 
bolus pressure during swallowing. Because manometry is not commonly performed in children [7], we did not carry out this evaluation. Recently, FEES has been more widely used in adults with dysphagia. In FEES, an endoscope is used to directly visualize the hypopharynx during the swallowing process to assess airway protection [5]. However, in our hospital, FEES is not routinely used for the assessment of swallowing disorders in children. If manometry and FEES had been performed, we could have more accurately determined the pathophysiology of dysphagia in this case.

\section{CONFLICT OF INTEREST}

No potential conflict of interest relevant to this article was reported.

\section{ACKNOWLEDGMENTS}

This research was supported by EMBRI Grants from the Eulji University (No. 2013-EMBRI-DJ0003).

\section{REFERENCES}

1. Posnick JC, Ruiz RL. Treacher Collins syndrome: current evaluation, treatment, and future directions. Cleft Palate Craniofac J 2000;37:434.

2. Dixon MJ. Treacher Collins syndrome. J Med Genet 1995;32:806-8.

3. Yokochi K, Terasawa S, Kono C, Fujishima I. Dysphagia in children with oculo-auriculo-vertebral spectrum. Dysphagia 1997;12:222-5.

4. Logemann JA. Manual for the videofluorographic study of swallowing. 2nd ed. Austin, TX: Pro-Ed; 1993.

5. Hiorns MP, Ryan MM. Current practice in paediatric videofluoroscopy. Pediatr Radiol 2006;36:911-9.

6. Liau JY, Sadove AM, van Aalst JA. An evidence-based approach to cleft palate repair. Plast Reconstr Surg 2010;126:2216-21.

7. Fung CW, Khong PL, To R, Goh W, Wong V. Video-fluoroscopic study of swallowing in children with neurodevelopmental disorders. Pediatr Int 2004;46:26-30.

8. Weckmueller J, Easterling C, Arvedson J. Preliminary temporal measurement analysis of normal oropharyngeal swallowing in infants and young children. Dysphagia 2011;26:135-43. 